\title{
ФАКТОРНЫЙ АНАЛИЗ ВЛИЯНИЯ ПАРАМЕТРОВ ВОДНОГО РЕЖИМА ПАСТБИЩ
}

(C) 2016

П.М. Мазуркин, доктор технических наук, профессор, заведующий кафедрой природообустройства, академик РАЕ и РАЕН, член Европейской Академии Естествознания ФГБОУ «Поволжский государственный технологический университет», Йошкар-Ола (Россия), kaf_po@mail.ru

Введение. Анализ бинарных отношений между 10 факторами проведен по данным [1, p.44-45] (Appendix C. Soil parameters used in the hydrological modelling). Статистическое моделирование выполнено идентификацией общей алгебраической формулы в виде суммы двух биотехнических законов [2-5].

Факторный анализ понимается как выявление устойчивых закономерностей изменений значений каждого из множества учитываемых параметров изучаемой системы, а также математических связей между факторами. В сравнении с аппроксимацией в методологии идентификации истинность устойчивых законов принимается как аксиома. Поэтому отпадает необходимость выбора эмпирической формулы - она заранее задана.

Наш метод факторного анализа позволяет не только устанавливать апостериорные причинно-следственные связи, но и давать им количественную характеристику, обеспечивает оценку уровня влияния факторов (влияющих параметров) на результаты функционирования (зависимые параметры). Это делает факторный анализ точным методом, а выводы - количественно обоснованными и осмысленными в ходе идентификации закономерностей.

Общая биотехническая закономерность. Индуктивно, на основе десятков тысяч примеров идентификации статистических выборок из различных областей науки, были выявлены две обобщенные математические модели [2-5]:

a) обобщенная детерминированная (трендовая) модель для идентификации по значениям факторов и связей между ними (показан в данной статье);

б) общая волновая функция колебательного возмущения изучаемой системы в виде асимметричного вейвлет-сигнала (предлагается по первичным не сгруппированным данным).

Все тенденции моделируются двухчленной закономерностью вида

$$
y=a_{1} x^{a_{2}} \exp \left(-a_{3} x^{a_{4}}\right)+a_{5} x^{a_{6}} \exp \left(-a_{7} x^{a_{8}}\right),
$$

где $y$ - оценочный параметр (параметр - это показатель изучаемой системы),

$x$ - влияющий параметр, в примере [1] 10 измеренных факторов по 20 значениям.

Исходные данные. Нами принимается допущение, что факторы исследователем выделены и соответствующая табличная модель (табл. 1) была составлена. Тогда факторный анализ - это выявление алгебраических связей между выделенными факторами.

Это и покажем на конкретном примере [1]. 
Таблица 1. Исходные данные [1] для статистического моделирования

\begin{tabular}{|c|c|c|c|c|c|}
\hline \multirow[t]{2}{*}{ Имя сайта } & $\begin{array}{c}\text { Topsoil hydraulic } \\
\text { conductivity } \\
\text { (m day) }\end{array}$ & $\begin{array}{c}\text { Subsoil hydrauli } \\
\text { conductivity } \\
\text { (m day) }\end{array}$ & $\begin{array}{l}\text { Topsoil } \\
\text { drainable } \\
\text { porosity }\end{array}$ & $\begin{array}{c}\text { Subsoil } \\
\text { drainable } \\
\text { pororsity }\end{array}$ & $\begin{array}{c}\text { Unsaturated Hy- } \\
\text { fraulic conductivit } \\
\text { exponent }\end{array}$ \\
\hline & $x_{1}$ & $x_{2}$ & $x_{3}$ & $x_{4}$ & $x_{5}$ \\
\hline 1. Belaugh & 3.0 & 3.0 & 0.3 & 0.3 & 8 \\
\hline 2. Blackthorn & 0.22 & 0.01 & 0.06 & 0.03 & 3 \\
\hline 3. Broaddale & 0.7 & 0.35 & 0.14 & 0.09 & 4 \\
\hline 4. Cricklade & 0.24 & 3.5 & 0.12 & 0.12 & 7 \\
\hline \multicolumn{6}{|l|}{ 5. Dancing Gate } \\
\hline 6. East Cottingwith & & - & & - & \\
\hline 7. East Harnham & 5.7 & & 0.11 & & 8 \\
\hline 8. Moorlinch & 0.6 & 0.6 & 0.16 & 0.16 & 8 \\
\hline 9. Mottey Meadows & 1 & & 0.13 & - & 8 \\
\hline 10. Nethercote & 0.41 & 0.73 & 0.1 & & \\
\hline 11. Portholme & 0.2 & 3.5 & 0.12 & 0.1 & 7 \\
\hline 12. Southlake & 0.08 & 1 & 0.12 & 0.14 & 7 \\
\hline 13. Stonygillfoot & 2.3 & 2.3 & 0.1 & 0.1 & 11 \\
\hline 14. Tadham & 2.5 & 1.75 & 0.15 & 0.15 & 8 \\
\hline 15. Tadham ESA & 2.5 & 1.75 & 0.15 & 0.15 & 8 \\
\hline 16. Upton Ham & 0.9 & 0.7 & 0.11 & 0.11 & 5 \\
\hline 17. Upwood & 0.22 & 0.01 & 0.06 & 0.02 & 3 \\
\hline 18. Westhay ESA & 2.5 & 1.75 & 0.15 & 0.15 & 8 \\
\hline 19. West Sedgemoor & 1.5 & 1.5 & 0.27 & 0.27 & 6.4 \\
\hline 20. Wet Moor & 0.1 & 3.35 & 0.06 & 0.15 & 8 \\
\hline
\end{tabular}

Продолжение таблицы 1

\begin{tabular}{|l|c|c|c|c|c|}
\hline \multirow{2}{*}{ Имя сайта } & $\begin{array}{c}\text { Rainfall } \\
(\mathrm{mm})\end{array}$ & $\begin{array}{c}\text { Potential transpi- } \\
\text { ration }(\mathrm{mm})\end{array}$ & $\begin{array}{c}\text { SMD (mm) } \\
(\text { end July) }\end{array}$ & $\begin{array}{c}\text { Prought thresh-1 } \\
\text { old }(\mathrm{cm})\end{array}$ & $\begin{array}{c}\text { Aeration } \\
\text { threshold }(\mathrm{cm})\end{array}$ \\
\cline { 2 - 6 } & $x_{6}$ & $x_{7}$ & $x_{8}$ & $x_{9}$ & $x_{10}$ \\
\hline 1. Belaugh & 575 & 531 & 107 & 49.4 & 35.7 \\
\hline 2. Blackthorn & 669 & 511 & 90 & 48.5 & 23.5 \\
\hline 3. Broaddale & 1663 & 375 & 0 & 47.7 & 30.4 \\
\hline 4. Cricklade & 726 & 503 & 82 & 44.6 & 34.1 \\
\hline 5. Dancing Gate & 1045 & 444 & 39 & 46.4 & 35.9 \\
\hline 6. East Cottingwith & 643 & 486 & 85 & - & - \\
\hline 7. East Harnham & 799 & 511 & 86 & 49.6 & 44.3 \\
\hline 8. Moorlinch & 865 & 523 & 85 & 46.8 & 27.3 \\
\hline 9. Mottey Meadows & 700 & 498 & 86 & 46.4 & 25.6 \\
\hline 10. Nethercote & 726 & 503 & 82 & 49.1 & 28.9 \\
\hline 11. Portholme & 574 & 523 & 103 & 48.3 & 38.7 \\
\hline 12. Southlake & 865 & 523 & 85 & 48.7 & 42.0 \\
\hline 13. Stonygillfoot & 1068 & 404 & 33 & 47.4 & 23.3 \\
\hline 14. Tadham & 865 & 523 & 85 & 48.8 & 35.6 \\
\hline 15. Tadham ESA & 865 & 523 & 85 & 48.8 & 35.6 \\
\hline 16. Upton Ham & 775 & 514 & 78 & 48.2 & 35.6 \\
\hline 17. Upwood & 574 & 523 & 103 & 48.5 & 23.5 \\
\hline 18. Westhay ESA & 865 & 523 & 85 & 48.8 & 35.6 \\
\hline 19. West Sedgemoor & 865 & 523 & 85 & 49.3 & 44.7 \\
\hline 20. Wet Moor & 865 & 523 & 85 & 49.3 & 42.7 \\
\hline
\end{tabular}


Из данных таблицы 1 видно, что они получены какой-то группировкой. Чаще всего группировку выполняют расчетом среднего арифметического значения. Если бы в нашем распоряжении были первичные данные измерений, то можно было бы выявить более точные статистические модели по 10 показателям с учетом волновых составляющих.

Рейтинг влияющих и зависимых факторов. В таблице 2 приведена полная корреляционная матрица (без ранговых распределений) бинарных (между парами взаимно влияющих факторов) связей между 10 факторами.

Таблица 2

Корреляционная матрица полного факторного анализа и рейтинг факторов

\begin{tabular}{|l|c|c|c|c|c|}
\hline \multirow{2}{*}{$\begin{array}{c}\text { Влияющие } \\
\text { факторы (параметры } x \text { ) }\end{array}$} & \multicolumn{4}{|c|}{ Зависимые факторы (показатели $y$ ) } \\
\cline { 2 - 6 } & $x_{1}$ & $x_{2}$ & $x_{3}$ & $x_{4}$ & $x_{5}$ \\
\hline Topsoil hydraulic conductivity (m day) $x_{1}$ & 1 & 0.5382 & 0.6028 & 0.6337 & 0.6409 \\
\hline Subsoil hydraulic conductivity (m day) $x_{2}$ & 0.8652 & 1 & 0.4973 & 0.6772 & 0.8634 \\
\hline Topsoil drainable porosity $x_{3}$ & 0.3666 & 0.2321 & 1 & 0.9079 & 0.4495 \\
\hline Subsoil drainable pororsity $x_{4}$ & 0.5910 & 0.5206 & 0.9293 & 1 & 0.7528 \\
\hline Unsaturated Hydraulic conductivity exponent $x_{5}$ & 0.6068 & 0.6989 & 0.4786 & 0.6994 & 1 \\
\hline Rainfall (mm) $x_{6}$ & 0.2550 & 0.2250 & 0.0739 & 0.2373 & 0.5864 \\
\hline Potential transpiration (mm) $x_{7}$ & 0.0176 & 0.1490 & 0.2092 & 0.3188 & 0.4963 \\
\hline SMD (mm) (end July) $x_{8}$ & 0.0167 & 0.2099 & 0.1194 & 0.2223 & 0.6612 \\
\hline Drought threshold (ст) $x_{9}$ & 0.5277 & 0.1634 & 0.1783 & 0.3824 & 0.0596 \\
\hline Aeration threshold (ст) $x_{10}$ & 0.3202 & 0.4632 & 0.3848 & 0.6131 & 0.2246 \\
\hline Cумма коэффициентов корреляции $\Sigma r$ & 4.5668 & 4.2003 & 4.4736 & 5.6921 & 5.7347 \\
\hline Место показателя $I_{y}$ & 8 & 10 & 9 & 5 & 4 \\
\hline
\end{tabular}

Продолжение таблицы 2

\begin{tabular}{|c|c|c|c|c|c|c|c|}
\hline \multirow{2}{*}{$\begin{array}{c}\text { Параметры } \\
x\end{array}$} & \multicolumn{3}{|c|}{ Зависимые факторы (показатели $y)$} & \multicolumn{2}{c|}{ Сумма } & Место \\
\cline { 2 - 6 } & $x_{6}$ & $x_{7}$ & $x_{8}$ & $x_{9}$ & $x_{10}$ & $\Sigma r$ & $I_{x}$ \\
\hline$x_{1}$ & 0.3702 & 0.3698 & 0.4575 & 0.5232 & 0.4469 & 5.5832 & 4 \\
\hline$x_{2}$ & 0.3517 & 0.3790 & 0.6024 & 0.6294 & 0.8915 & 6.7571 & $\mathbf{1}$ \\
\hline$x_{3}$ & 0.0033 & 0.3565 & 0.1194 & 0.4246 & 0.3474 & 4.2073 & 9 \\
\hline$x_{4}$ & 0.3726 & 0.6964 & 0.6342 & 0.4903 & 0.7067 & 6.6939 & $\mathbf{2}$ \\
\hline$x_{5}$ & 0.2785 & 0.6658 & 0.5374 & 0.1581 & 0.7606 & 5.8841 & $\mathbf{3}$ \\
\hline$x_{6}$ & 1 & 0.9021 & 0.9525 & 0.1825 & 0.4483 & 4.8630 & 7 \\
\hline$x_{7}$ & 0.8767 & 1 & 0.9514 & 0.5864 & 0.3902 & 4.9956 & 6 \\
\hline$x_{8}$ & 0.9363 & 0.9683 & 1 & 0.4164 & 0.9553 & 5.5058 & 5 \\
\hline$x_{9}$ & 0.2545 & 0.5205 & 0.4263 & 1 & 0.6013 & 4.1140 & 10 \\
\hline$x_{10}$ & 0.3716 & 0.3828 & 0.3417 & 0.5029 & 1 & 4.6049 & 8 \\
\hline$\Sigma r$ & 4.8154 & 6.2412 & 6.0228 & 4.9138 & 6.5482 & 53.2089 & - \\
\hline Место $I_{y}$ & 7 & $\mathbf{2}$ & $\mathbf{3}$ & 6 & $\mathbf{1}$ & - & 0.5321 \\
\hline
\end{tabular}

В таблице 3 даны компактные записи значений параметров модели (1). 
Таблица 3. Параметры закономерностей взаимного влияния показателей

\begin{tabular}{|c|c|c|c|c|c|c|c|c|c|c|}
\hline \multirow{2}{*}{\multicolumn{2}{|c|}{ Факторы }} & \multicolumn{8}{|c|}{ Общий закон $y=a_{1} x^{a_{2}} \exp \left(-a_{3} x^{a_{4}}\right)+a_{5} x^{a_{6}} \exp \left(-a_{7} x^{a_{8}}\right)$} & \multirow{3}{*}{$\begin{array}{c}\text { Коэф. } \\
\text { корр. } \\
r\end{array}$} \\
\hline & & \multicolumn{4}{|c|}{ Первая составляющая } & \multicolumn{4}{|c|}{ Вторая составляющая } & \\
\hline$x$ & $y$ & $a_{1}$ & $a_{2}$ & $a_{3}$ & $a_{4}$ & $a_{5}$ & $a_{6}$ & $a_{7}$ & $a_{8}$ & \\
\hline$x_{8}$ & $x_{7}$ & 374.70374 & 0 & 0.026208 & 1 & $6.51818 \mathrm{e} 6$ & 5.08560 & 16.64811 & 0.14794 & 0.9683 \\
\hline$x_{8}$ & $x_{10}$ & $3.22802 \mathrm{e} 7$ & 8.18164 & 23.41995 & 0.17105 & 0 & 0 & 0 & 0 & 0.9553 \\
\hline$x_{6}$ & $x_{8}$ & 40.23982 & 0 & -0.0023136 & 1 & $-4.55658 \mathrm{e}-8$ & 3.29629 & 0 & 0 & 0.9525 \\
\hline$x_{7}$ & $x_{8}$ & $-1.14232 \mathrm{e} 7$ & 0 & 1.53724 & 0.34910 & 0.0044124 & 1.60871 & 0 & 0 & 0.9514 \\
\hline$x_{8}$ & $x_{6}$ & 1652.6879 & 0 & 0.028053 & 0.74615 & 0 & 0 & 0 & 0 & 0.9363 \\
\hline$x_{4}$ & $x_{3}$ & 0.057820 & 0 & -5.07272 & 0.92808 & 0 & 0 & 0 & 0 & 0.9293 \\
\hline$x_{3}$ & $x_{4}$ & 0.033043 & 0 & 0 & 0 & 1.26462 & 1.28611 & 0 & 0 & 0.9079 \\
\hline$x_{6}$ & $x_{7}$ & 227.60211 & 0 & -0.0020124 & 1 & $-5.09798 \mathrm{e}-7$ & 3.12871 & 0 & 0 & 0.9021 \\
\hline$x_{2}$ & $x_{10}$ & 23.75135 & 0 & -0.017314 & 2.67978 & 17.15156 & 1.95212 & 0.051754 & 6.18305 & 0.8915 \\
\hline$x_{7}$ & $x_{6}$ & 386020.2 & 0 & 0.0078186 & 0.94425 & -6774.0186 & 0.83325 & 0.014992 & 0.89617 & 0.8767 \\
\hline$x_{2}$ & $x_{1}$ & 0.49583 & 0 & 0.45055 & 1 & 39.61873 & 12.39564 & 5.60147 & 1.00944 & 0.8652 \\
\hline$x_{2}$ & $x_{5}$ & 3.02871 & 0 & 0.37467 & 1 & 7.85863 & 1.18715 & 0.47018 & 1 & 0.8634 \\
\hline$x_{5}$ & $x_{10}$ & 4.28454 & 2.38270 & 0.27272 & 1.12590 & 0 & 0 & 0 & 0 & 0.7606 \\
\hline$x_{4}$ & $x_{5}$ & 2.79236 & 0 & -3.31653 & 18.59787 & $1.75307 \mathrm{e} 8$ & 4.77904 & 23.86727 & 0.55696 & 0.7528 \\
\hline$x_{4}$ & $x_{10}$ & 23.48359 & 0 & 8.22510 & 1 & 996.42298 & 1.43515 & 5.22215 & 1 & 0.7067 \\
\hline
\end{tabular}

Коэффициент функциональной связности (коррелятивной вариации) 10 факторов равен 53.2089 / $10^{2}=0.5321$. Этот критерий применяется при сопоставлении разных фитоценозов, в частности, пастбищ из разных регионов.

По таблице 2 среди влияющих факторов первое место занял $x_{2}$, второе $x_{4}$ и третье $-x_{5}$. Среди зависимых факторов на первое место вышел фактор Aeration threshold, а второе - Potential transpiration и третье - SMD end July.

Корреляционная матрица бинарных отношений. Из данных таблицы 2 исключим рейтинг и клетки с единицами по диагонали (табл. 4).

Таблица 4

Корреляционная матрица бинарных отношений между факторами

\begin{tabular}{|c|c|c|c|c|c|c|c|c|c|c|}
\hline & $x_{1}$ & $x_{2}$ & $x_{3}$ & $x_{4}$ & $x_{5}$ & $x_{6}$ & $x_{7}$ & $x_{8}$ & $x_{9}$ & $x_{10}$ \\
\hline$x_{1}$ & & 0.5382 & 0.6028 & 0.6337 & 0.6409 & 0.3702 & 0.3698 & 0.4575 & 0.5232 & 0.4469 \\
\hline$x_{2}$ & 0.8652 & & 0.4973 & 0.6772 & 0.8634 & 0.3517 & 0.3790 & 0.6024 & 0.6294 & 0.8915 \\
\hline$x_{3}$ & 0.3666 & 0.2321 & & 0.9079 & 0.4495 & $\mathbf{0 . 0 0 3 3}$ & 0.3565 & 0.1194 & 0.4246 & 0.3474 \\
\hline$x_{4}$ & 0.5910 & 0.5206 & 0.9293 & & 0.7528 & 0.3726 & 0.6964 & 0.6342 & 0.4903 & 0.7067 \\
\hline$x_{5}$ & 0.6068 & 0.6989 & 0.4786 & 0.6994 & & 0.2785 & 0.6658 & 0.5374 & 0.1581 & 0.7606 \\
\hline$x_{6}$ & 0.2550 & 0.2250 & 0.0739 & 0.2373 & 0.5864 & & 0.9021 & 0.9525 & 0.1825 & 0.4483 \\
\hline$x_{7}$ & 0.0176 & 0.1490 & 0.2092 & 0.3188 & 0.4963 & 0.8767 & & 0.9514 & 0.5864 & 0.3902 \\
\hline$x_{8}$ & 0.0167 & 0.2099 & 0.1194 & 0.2223 & 0.6612 & 0.9363 & $\mathbf{0 . 9 6 8 3}$ & & 0.4164 & 0.9553 \\
\hline$x_{9}$ & 0.5277 & 0.1634 & 0.1783 & 0.3824 & 0.0596 & 0.2545 & 0.5205 & 0.4263 & & 0.6013 \\
\hline$x_{10}$ & 0.3202 & 0.4632 & 0.3848 & 0.6131 & 0.2246 & 0.3716 & 0.3828 & 0.3417 & 0.5029 & \\
\hline
\end{tabular}


Тогда получим множество коэффициентов корреляции у бинарных связей. Максимальная адекватность 0.9683 наблюдается у $x_{7}=f\left(x_{8}\right)$. Минимальный коэффициент корреляции 0.0033 равен для отношения $x_{6}=f\left(x_{3}\right)$.

Выше известного уровня адекватности 0.7 имеются 15 бинарных связей (табл. 4), что составляет $100 \times 15 /\left(10^{2}-10\right)=16.67 \%$. При этом доля сильнейших бинарных отношений очень далека от золотой пропорции $61.80 \%$.

Серым цветом выделен блок кластерного анализа из парных связей между тремя факторами $x_{6}, x_{7}$ и $x_{8}$. Образовалось три кластера закономерностей.

Первый кластер. Он обладает хорошей симметрией (табл. 5).

Таблица 5. Первый кластер

сильнейших связей

\begin{tabular}{|c|c|c|c|}
\hline & $x_{6}$ & $x_{7}$ & $x_{8}$ \\
\hline$x_{6}$ & & 0.9021 & 0.9525 \\
\hline$x_{7}$ & 0.8767 & & 0.9514 \\
\hline$x_{8}$ & 0.9363 & 0.9683 & \\
\hline
\end{tabular}

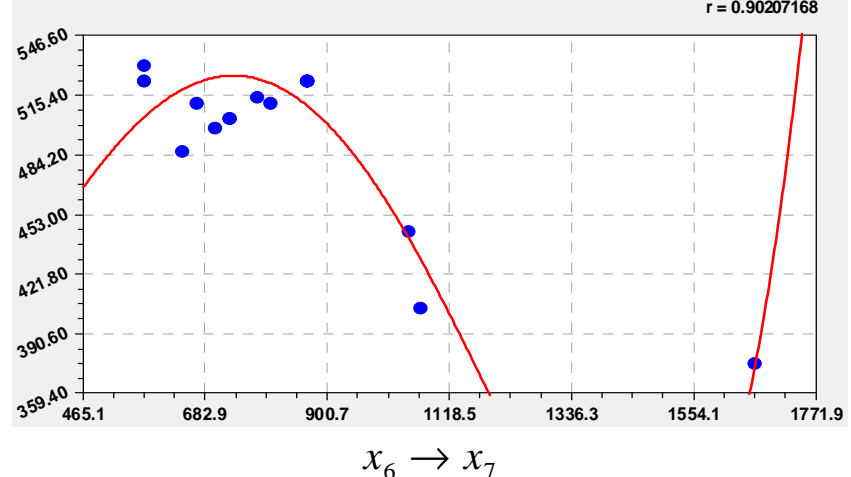
нические закономерности. По сути три фактора $x_{6}$ (Rainfall), $x_{7}$ (Potential transpiration) и $x_{8}$ (SMD end July) становятся неким ядром для всего множества факторов.

Графики показаны на рисунке 1.

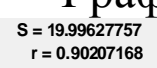
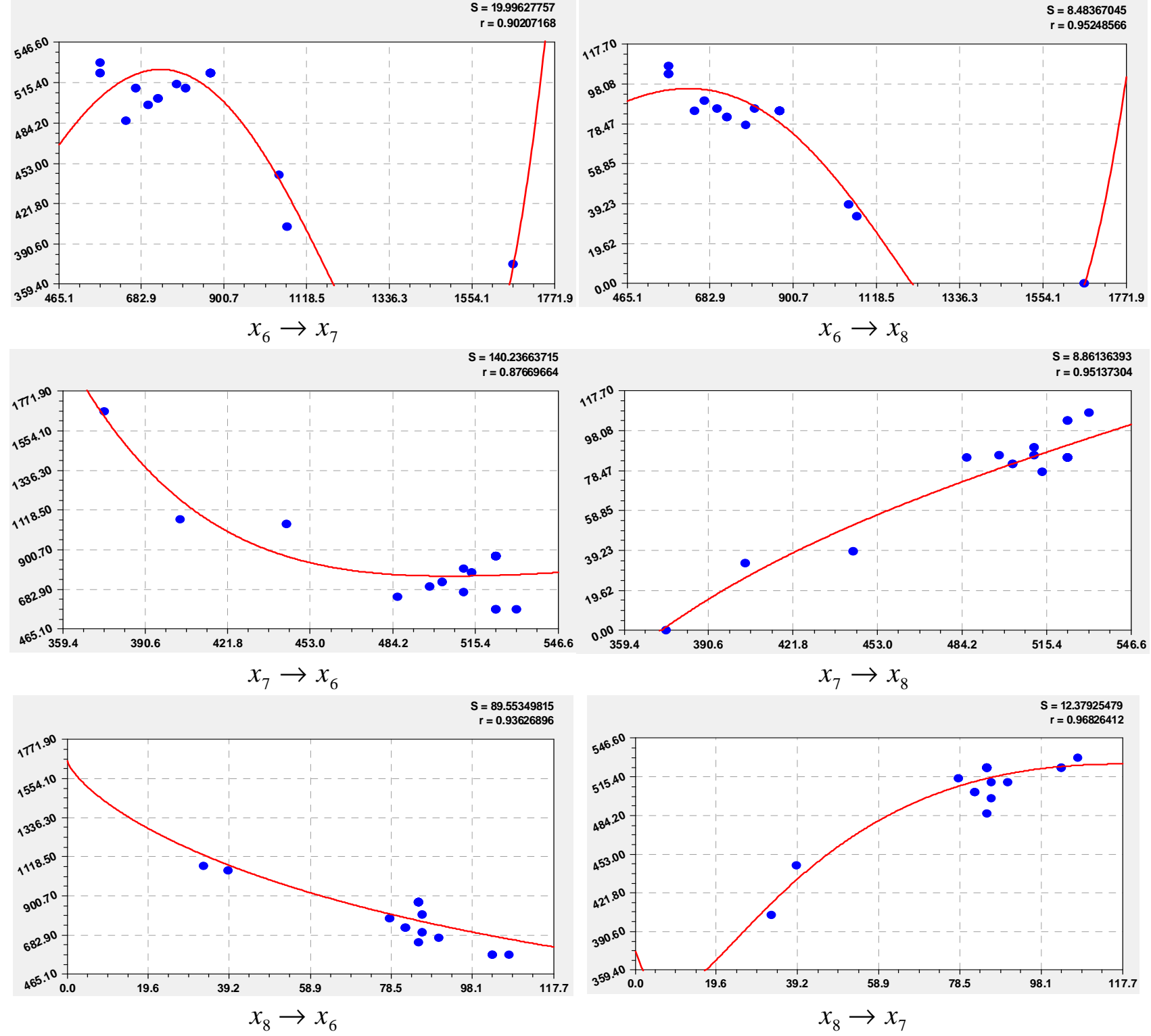

Рис. 1. Графики бинарных отношений первого кластера закономерностей 
Из-за повторов значений из 20 имён сайтов образовались всего 12 точек. Как видно из рисунка 1, эти 12 точек образуют большой кластер исходных данных из девяти точек, расположенных близко друг к другу. Таким образом, кластеризация происходит не только по закономерностям, но и по значениям факторов, входящих в эти биотехнические закономерности. В итоге при использовании всех первичных данных появятся и волновые возмущения.

Второй кластер. Он определился (табл. 6) влиянием на показатель $x_{10}$ (Aeration threshold) четырех влияющих переменных $x_{2}$ (Subsoil hydraulic conductivity), $x_{4}$ (Subsoil drainable pororsity), $x_{5}$ (Unsaturated Hydraulic conductivity exponent) и $x_{8}$ (SMD end July).

На рисунке 2 показаны графики бинарных отношений. Коэффициент корреляции $r$ дан в правом верхнем углу каждого графика.

Таблица 6. Вто-
рой кластер
сильных связей
\begin{tabular}{|c|c|}
\hline & $x_{10}$ \\
\hline$x_{2}$ & 0.8915 \\
\hline$x_{4}$ & 0.7067 \\
\hline$x_{5}$ & 0.7606 \\
\hline$x_{8}$ & 0.9553 \\
\hline
\end{tabular}
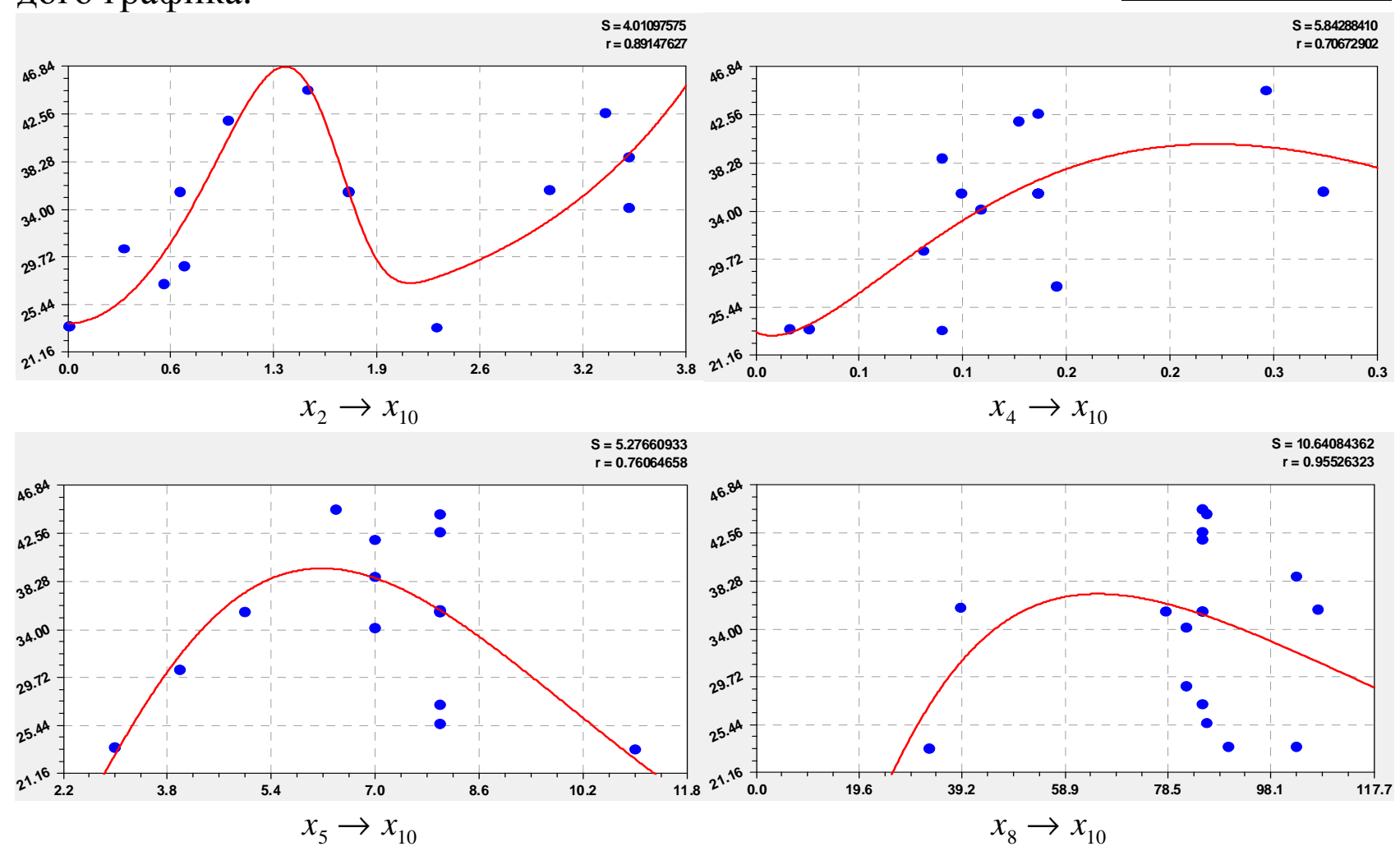

Рис. 2. Графики бинарных отношений второго кластера закономерностей

Третий кластер (табл. 7) получен дополнением к группе закономерностей из таблицы 4 фактора $x_{5}$ (Unsaturated Hydraulic conductivity exponent).

Таблица 7. Третий кластер бинаров

\begin{tabular}{|c|c|c|c|c|c|}
\hline & $x_{1}$ & $x_{2}$ & $x_{3}$ & $x_{4}$ & $x_{5}$ \\
\hline$x_{2}$ & 0.8652 & & & & 0.8634 \\
\hline$x_{3}$ & & & & 0.9079 & \\
\hline$x_{4}$ & & & 0.9293 & & 0.7528 \\
\hline$x_{5}$ & 0.6068 & 0.6989 & & 0.6994 & \\
\hline
\end{tabular}

Это влияние на три показателя $x_{1}$ (Topsoil hydraulic conductivity), $x_{2}$ (Subsoil hydraulic conductivity) и $x_{4}$ (Subsoil drainable pororsity) происходит с коэффициентами корреляции менее 0,7 .

На рисунке 3 даны графики восьми бинарных отношений. В дополнение к таблице 5 биотехническое влияние происходит по формулам: 


$$
\begin{aligned}
x_{4} & =0.00089702 x_{5}{ }^{5.30108} \exp \left(-0.72580 x_{5}{ }^{0.99904}\right) ; \\
x_{2}= & 9.97568 \cdot 10^{7} x_{5}^{18.18652} \exp \left(-28.52592 x_{5}{ }^{0.31926}\right) ; \\
x_{1} & =3.11204 \cdot 10^{-6} x_{5}{ }^{20.53199} \exp \left(-4.05046 x_{5}\right)+ \\
& +1.55852 \cdot 10^{-48} x_{5}^{89.91067} \exp \left(-9.51579 x_{5}\right)
\end{aligned}
$$
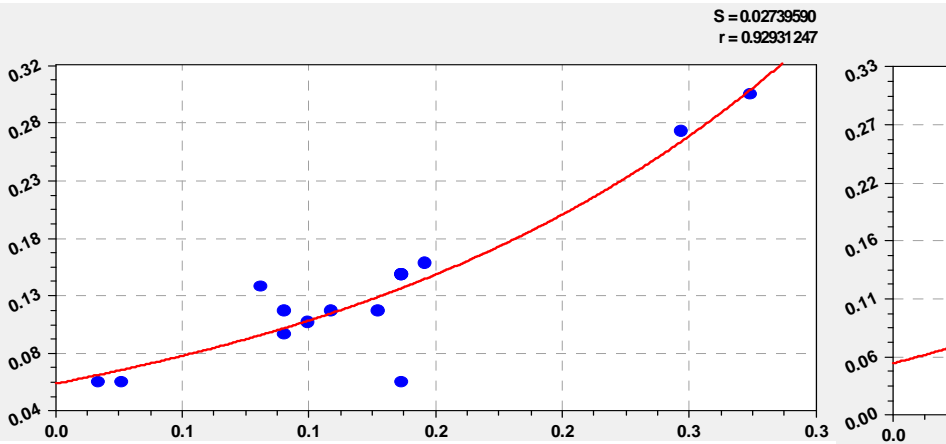

$$
x_{4} \rightarrow x_{3}
$$
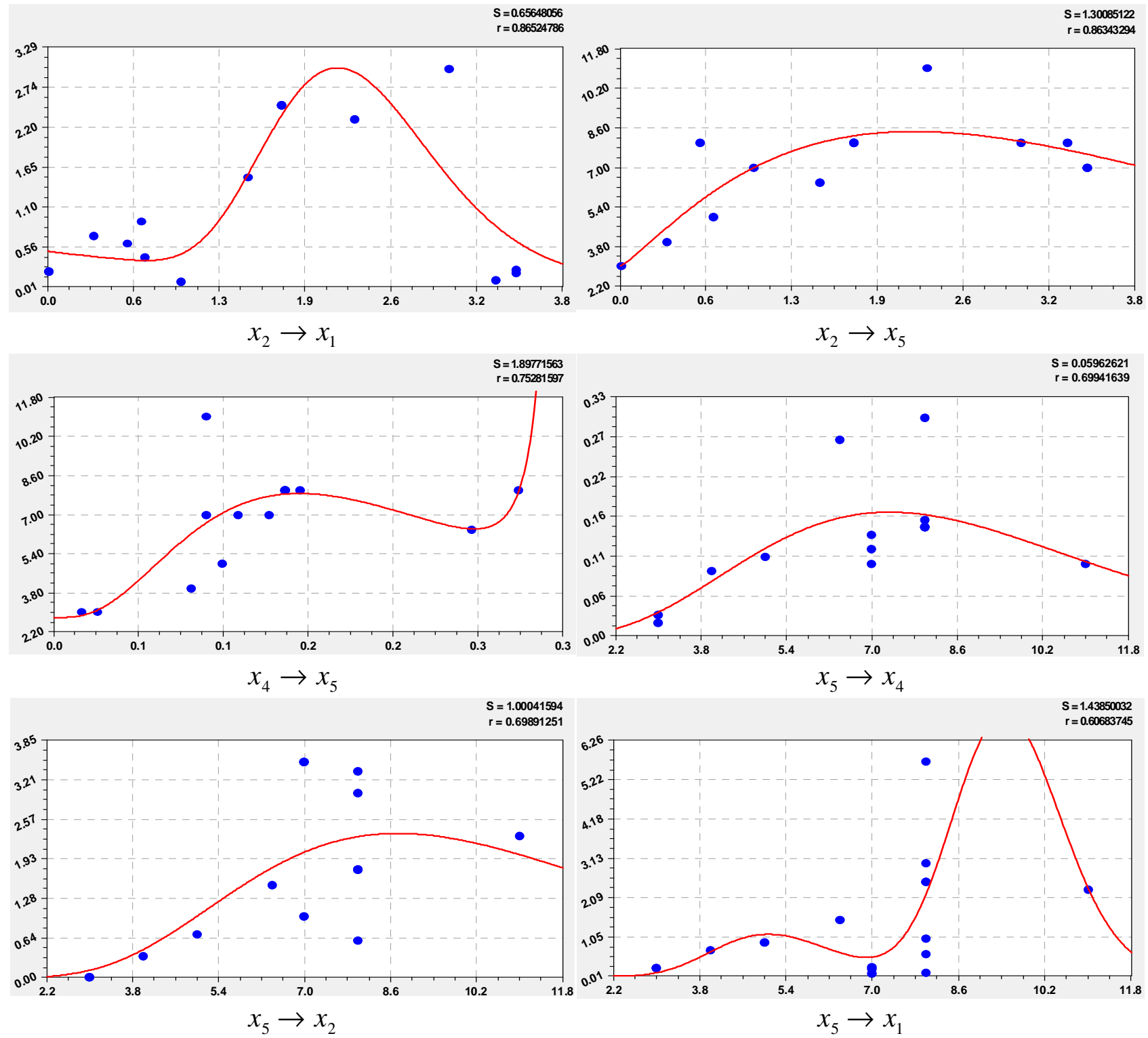

Рис. 3. Графики бинарных отношений третьего кластера закономерностей 
закономерностей сильно меняется, поэтому получаются различающиеся по сложности графики.

Заключение. Между биохимическими веществами почвы пастбищ и гидрологическими параметрами водного режима всегда существует гомеостаз.

По общей табличной модели [1], после выявления сильных факторных связей, вполне можно определить оптимальные или рациональные значения у всех 10 факторов. А затем можно по статистическим моделям прогнозировать продуктивность сенокосов и пастбищ по рациональным значениям факторов.

После этого можно будет приступать к параметрическому обоснованию мероприятий по улучшению водного режима прирусловых территорий. Это позволит осознано значительно повысить продуктивность пойменных лугов.

\section{СПИСОК ЛИТЕРАТУРЫ}

1. The Water Regime Requirements and the Response to Hydrological Change of Grassland Plant Communities. DEFRA-commissioned project BD1310. Final report to the Department for Environment, Food and Rural Affairs. Institute of Water and Environment. Silsoe. Bedford. November 2002. 98 p.

2. Mazurkin P.M. Method of identification // $14^{\text {th }}$ International multidisciplinary scientific geoconferenct \& SGEM2014. GeoConference jn NANO, BIO AND GREEN - TECHNOLOGIES FOR A SUSTAINABLE FUTURE. Conference proceedincs. Volume 1. Section Advances in Biotechnology. 17-26 June 2014. Albena, Bulgaria. P. 427-434.

3. Mazurkin P.M.. Factor analysis of the technical level of sibgle-bucket hydraulic excavators // International Journal of Engineering and Innovative Technology (IJEIT), Volume 3, Issue 9, March 2014. p.84-92.

4. Mazurkin P.M.. Statistical modeling of entire prime numbers / International Journal of Engineering and Technical Research (IJETR) ISSN: 2321-0869, Volume2, Issue-8, August 2014. P.148-158.

5. Mazurkin P.M., Tishin D.V. Wave dynamics of tree-ring width jf Oak // Integrated Journal of British. Volume 2. 2015. Issue 1. JAN-FEB. P. 55-67. IJBRITISH-223-PA.pdf.

\section{FACTOR ANALYSIS OF PARAMETERS WATER REGIME PASTURES} (C) 2016

P.M. Mazurkin, Doctor of Technical Sciences, Professor, Academician of the Russian Academy of Natural Sciences and the Russian Academy of Natural Sciences, Member of the European Academy of Natural Sciences,

Volga State Technological University (Yoshkar-Ola), Russia, kaf_po@mail.ru 